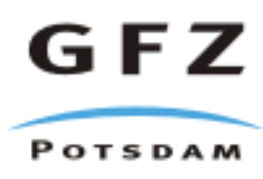

Originally published as:

Greiner-Mai, H., Hagedoorn, J., Ballani, L., Wardinski, I., Stromeyer, D., Hengst, R. (2007): Axial poloidal electromagnetic core-mantle coupling torque: a re-examination for different conductivity and satellite supported geomagnetic field models. - Studia Geophysica et Geodaetica, 51, 4, 491-513

DOI: 10.1007/s11200-007-0029-0. 


\title{
Axial poloidal electromagnetic core-mantle coupling torque: a re-examination for different conductivity and satellite supported geomagnetic field models
}

\author{
H. Greiner-Mai ${ }^{1}$, J. Hagedoorn ${ }^{1}$, L. Ballani ${ }^{1}$, I. Wardinski ${ }^{2}$, D. Stromeyer ${ }^{3}$, \\ R. $\operatorname{HenGST}^{1}$
}

1 GeoForschungsZentrum Potsdam, Sec. 1.5 Earthsystem Modelling, Telegrafenberg, 14473 Potsdam, Germany (grm@gfz-potsdam.de)

2 GeoForschungsZentrum Potsdam, Sec. 2.3 Earth's Magnetic Field, Telegrafenberg, 14473 Potsdam, Germany

3 GeoForschungsZentrum Potsdam, Sec. 5.3 Engineering Seismology, Telegrafenberg, 14473 Potsdam, Germany

\begin{abstract}
We investigate the temporal behaviour of the axial component of the electromagnetic coremantle coupling torque that is associated with the poloidal part of the geomagnetic field observable at the Earth surface. For its computation, we use different models of the geomagnetic field, expanded into spherical harmonics (Wardinski and Holme, 2006; Sabaka et al., 2004), and the mantle conductivity. The geomagnetic field, which we have to know at the coremantle boundary for the associated computations, will be inferred from the field at the Earth surface by the non-harmonic field continuation through a conducting mantle shell. The aims of this investigation are (i) to check how sensitive is the computation of the torque with respect to the different geomagnetic field models, (ii) to check its dependence on the spherical harmonic degree $n$, and (iii) to determine the difference between the mechanical torque derived from the observed length-of-day variations (atmospheric influence subtracted) and the poloidal electromagnetic torque in dependence on the assumed conductivity. To use the non-harmonic field continuation for the torque calculation and to obtain an insight into the influence of the different geomagnetic field models on the EM torques are the major aspects of this paper.
\end{abstract}

\section{INTRODUCTION}

The observed decadal variations of the length of day ( $\Delta \mathrm{LOD})$ are usually attributed to the exchange of angular momentum between the fluid core and solid mantle. This has been concluded from the observed correlations between geomagnetic variations and $\triangle$ LOD, and from the small effect of surface processes on this time scale (e.g. Greiner-Mai and Jochmann (1998) show that the atmosphere can only be responsible for $14 \%$ of decadal $\Delta$ LOD). Since several decades, various core-mantle coupling processes have been probed to explain $\Delta \mathrm{LOD}$, from which the electromagnetic (EM) coupling (e.g. Rochester, 1960; Roberts, 1972; Stix and Roberts, 1984; Greiner-Mai, 1987, 1993; Holme, 1998a,b) is partially re-examined in this paper. The EM torques can reach orders of magnitude, which are sufficient to explain 
$\Delta$ LOD if the assumed mantle conductivity, respective conductance, is sufficiently high. EM torques are produced by two processes: (i) temporal variations of the geomagnetic field induce electric currents in the conducting part of the mantle, and (ii) currents produced in the core leak into the mantle. The currents in the mantle produce a Lorentz force on it by their interaction with the geomagnetic field.

To calculate the EM coupling torques, it is necessary to know the electric conductivity, $\sigma_{M}$, of the mantle, and the geomagnetic field within it. In Section 3 we will show that the torque computation can be reduced to an integration over the core-mantle boundary (CMB). This means that we finally must know the geomagnetic field at the CMB. Because a non-zero conductivity is a precondition for the existence of electric currents, we have to determine the geomagnetic field at the CMB from its observed values at the Earth's surface by a solution of the induction equation of the mantle. Recently, Ballani et al. (2002) developed an algorithm for a rigorous inversion of the mantle induction equation to obtain the poloidal geomagnetic field at the $\mathrm{CMB}$, and checked this method also for highly conducting core shells. In this paper, we use this so-called non-harmonic downward continuation (NHDC) instead of the perturbation method of Benton and Whaler (1983) generally used before. This is one of the motivations to re-calculate axial EM torques. The application of the NHDC and the formalism in its background (see Section 2 and 3) has another advantage. We do not need in addition to the geomagnetic field at the CMB the related time derivations for the calculation of the EM torque. The computation of the time derivatives would be associated with additional uncertainties.

In previous investigations of the EM coupling, it has been implied that the calculated torques are mainly determined by the low degrees $n$ of the spherical harmonic (SH) expansions of the geomagnetic field at any time and are not sensitive to the available geomagnetic models. The existence of two new satellite supported highly resolved geomagnetic field models (Wardinski and Holme, 2006; Sabaka et al., 2004) for the period 1980 to 2000 gives us the possibility, to investigate the dependence of the torque on the geomagnetic field models and on the SH degree $n$ of this models, respectively. This is the second motivation for the investigations in this paper. A first check of this dependence was given by Stix (1982) for the example of a field model of the year 1975 for SH expansions with summations over $n$ up to $1,6,12$. Here, we will extend his investigation with respect to the poloidal part of the axial torque to (i) check its behavior for each point of the time series between 1982 and 1998, and (ii) compute the torque for summations up to $1,2,3, \ldots, n_{\max }$ (i.e. in steps of 1 ).

The investigation presented here can only be considered as a first step towards a consistent description of EM core-mantle coupling. In terms of a minimum effort, we use for these investigations the axial part of the poloidal EM torque to study the effect of the conductivity assumptions (Section 4) with the NHDC of the geomagnetic field, and to study the dependence of the torque on the considered $\mathrm{SH}$ degree of the geomagnetic field models to explore the significance of the higher SH degrees for the EM torque computation.

This approach is similar in some respect to that of Paulus and Stix (1989), like splitting of the geomagnetic field into poloidal and toroidal part and the restriction to the poloidal part.

The assumptions about the conductivity models in Section 4 are just made for the same pragmatical reasons. They should only ensure that one of them has a conductance which is approximately consistent with EM coupling according to Holme (1998b).

Despite of these two major objectives, we calculate the torques necessary to produce the 
observed residuals of $\triangle \mathrm{LOD}$ obtained after subtraction of the atmospheric contribution, and compute the difference between this mechanical and the EM torque. This way we will check the temporal structure of the difference as a characteristic curve of the sum of the remaining torques to be modelled.

\section{THE NON-HARMONIC FIELD CONTINUATION TO THE CMB}

The electromagnetic torque, $\mathbf{L}$, results from Lorentz forces on the mantle caused by the interaction of electric currents (density $\mathbf{j}$ ) with the magnetic field (flux $\mathbf{B}$ ). It is defined by

$$
\mathbf{L}=\int_{V_{M}} \mathbf{r} \times(\mathbf{j} \times \mathbf{B}) \mathrm{d} V,
$$

where $V_{M}$ is the volume of the conducting part of the mantle, and $\mathbf{j} \times \mathbf{B}$ is the density of the Lorentz forces. A net EM torque can only be produced, if $\mathbf{j} \neq 0$, i.e. a significant mantle conductivity exists. This means that the induction equation must be solved to infer the necessary magnetic flux in the conducting mantle volume and/or at the CMB from its values at the Earth's surface. Therefore, the field continuation to the CMB must in any case be non-harmonic. In this paper, we will apply the recently developed non-harmonic downward continuation (NHDC) of the geomagnetic field by a rigorous inversion of the induction equation according to Ballani et al. (2002), to compute the geomagnetic field at the CMB necessary to calculate the poloidal torque. We will outline this inversion in the following two sections.

\subsection{THE MANTLE INDUCTION EQUATION}

The induction equation of the mantle is conventionally derived from Maxwell equations (displacement currents neglected) and Ohm's law by eliminating $\mathbf{j}$ and the electric field, to reduce the involved number of fields to the magnetic flux, B. We obtain the following vectorial form of the induction equation

$$
\operatorname{curl}\left(\frac{1}{\mu_{0} \sigma_{M}(r)} \operatorname{curl} \mathbf{B}\right)=-\dot{\mathbf{B}},
$$

where $\mu_{0}$ is the permeability of vacuum, and $\sigma_{M}(r)$ is the electrical mantle conductivity, assumed to be dependent on the radius, $r$, only.

To transform the vectorial induction equation into a scalar form, we divide $\mathbf{B}$ in its poloidal and toroidal parts. Because of $\operatorname{div} \mathbf{B}=0, \mathbf{B}$ can be represented by two defining scalar functions $S(r, \vartheta, \varphi, t)$ and $T(r, \vartheta, \varphi, t)$ (instead of three) as follows

$$
\mathbf{B}=\mathbf{B}_{t}+\mathbf{B}_{p}=\operatorname{curl}(\mathbf{r} T)+\operatorname{curl} \operatorname{curl}(\mathbf{r} S) .
$$

By known formula of vector analysis, we can express these field parts by

$$
\begin{aligned}
& \mathbf{B}_{p}=-\mathbf{r} \Delta S+\nabla \tilde{S}, \quad \tilde{S}=\frac{\partial}{\partial r}(r S), \\
& \mathbf{B}_{t}=-\mathbf{r} \times \nabla T
\end{aligned}
$$


where $\Delta$ and $\nabla$ are the Laplacean and the Nabla operators, respectively. From eq. (5) we can conclude that the radial component, $B_{r}$, appears only in the poloidal field. Using eq. (3) and some rules for the toroidal and poloidal fields (e.g. Krause and Rädler, 1980, Chap. 13; Gubbins and Roberts, 1987, Sec. 3.2), we obtain two scalar induction equations

$$
\begin{array}{ll}
\Delta S & =\mu_{0} \sigma_{M}(r) \dot{S}, \\
\Delta T-\frac{1}{\sigma_{M}(r) \cdot r} \frac{\mathrm{d} \sigma_{M}(r)}{\mathrm{d} r} \frac{\partial}{\partial r}(r T) & =\mu_{0} \sigma_{M}(r) \dot{T} .
\end{array}
$$

The poloidal field can completely be inferred from the observed part of the geomagnetic field by inversion of eq. (6), whereas the toroidal field vanishes outside of a conductor and therefore can only be determined with additional information. Typically this information is extracted from core-flow models. Because the induction equations of the poloidal and the toroidal fields are decoupled for a radially symmetric conductivity according to eqs. (6) and (7), we do not need the toroidal field to evaluate the poloidal torque, which is the subject of this paper. This would not be the case if we would assume a laterally heterogeneous conductivity distribution (e.g. Holme, 2000).

The scalar function $S$ can be expanded (for any fixed radius $r$ ) into a series of surface spherical harmonic (SH) functions, $\left\{P_{n m}(\cos \vartheta) \cos m \varphi, P_{n m}(\cos \vartheta) \sin m \varphi\right\}$, by

$$
S(r, \vartheta, \varphi, t)=\sum_{n=1}^{n_{\max }} \sum_{m=0}^{n}\left[S_{n m}^{c}(r, t) \cos m \varphi+S_{n m}^{s}(r, t) \sin m \varphi\right] P_{n m}(\cos \vartheta),
$$

where the normalization (Ferrers-Neumann)

$$
\int_{0}^{\pi}\left[P_{n m}(\cos \vartheta)\right]^{2} \sin \vartheta \mathrm{d} \vartheta=\frac{2}{2 n+1} \frac{(n+m) !}{(n-m) !}
$$

is used here. If the operator

$$
\Omega=\frac{1}{\sin \vartheta} \frac{\partial}{\partial \vartheta}\left(\sin \vartheta \frac{\partial}{\partial \vartheta}\right)+\frac{1}{\sin ^{2} \vartheta} \frac{\partial^{2}}{\partial \varphi^{2}}
$$

means the Laplacean on the sphere the eigenvalue equation

$$
\Omega\left(P_{n m}(\cos \vartheta)\left\{\begin{array}{c}
\cos m \varphi \\
\sin m \varphi
\end{array}\right\}\right)=-n(n+1) P_{n m}(\cos \vartheta)\left\{\begin{array}{c}
\cos m \varphi \\
\sin m \varphi
\end{array}\right\}
$$

holds. With these relations and eq. (6), we can find the induction equation for single SH modes of $S$

$$
\frac{\partial^{2} S_{n m}^{c, s}}{\partial r^{2}}+\frac{2}{r} \frac{\partial S_{n m}^{c, s}}{\partial r}-\frac{n(n+1)}{r^{2}} S_{n m}^{c, s}=\mu_{0} \sigma_{M}(r) \dot{S}_{n m}^{c, s} .
$$

The boundary conditions are given by

$$
S_{n m}^{c, s}\left(R_{\sigma}, t\right)=\Phi_{n m}^{c, s}(t), \quad \frac{\partial S_{n m}^{c, s}\left(R_{\sigma}, t\right)}{\partial r}+\frac{n+1}{R_{\sigma}} S_{n m}^{c, s}\left(R_{\sigma}, t\right)=0,
$$

where $R_{\sigma}$ is the radius of the spherical interface between the conducting and the nonconducting part of the mantle (see fig. 1). $\Phi_{n m}^{c, s}$ are the boundary values derived by harmonic continuation of the coefficients of the geomagnetic surface potential field to this boundary at $r=R_{\sigma}$. The derivation is given in the Appendix. 


\subsection{INVERSION OF THE INDUCTION EQUATION - AN OUTLINE}

The details of the theory of the inversion method, the used numerical algorithm and the results of some checks of the method for highly conducting shells are given in Ballani et al. (2002) and Greiner-Mai et al. (2004). Here, we will give an outline to make it understandable how the method works without going too much into details.

With eqs. (12) and (13) an initial-boundary value problem can be formulated. Like for the analogously structured well-studied problems in the inverse heat conduction theory, the boundary values are given only on one side of the radial interval, here at $r=R_{\sigma}$ (upper mantle side, see fig. 1). This problem is temporally unstable towards the higher-frequency part and, in addition, it suffers from the wide range of order of magnitudes. However, uniqueness holds for the solution under relatively general assumptions. The geomagnetic potential $V(r, \vartheta, \varphi, t)(\mathbf{B}=-\operatorname{grad} V$, see eq. (33) in the Appendix), determined at the Earth's surface, can be harmonically continued to $R_{\sigma}$, obtaining the boundary values $\Phi_{n m}^{c, s}(t)$ in eq. (13).

The initial condition can be chosen arbitrarily. But its influence on the solution is mainly limited to the beginning of the time interval, e.g. 2-3 years are observed in our cases. In addition, the regularization gives no real information towards the interval end. Therefore, we reduce the time interval for the EM torque to $1982-1998$. One possible choice for the initial condition is that of the harmonically continued $\mathbf{B}$-field for $R_{\sigma} \geq r \geq R_{c}$.

The solution of the downward continuation problem (12) and (13) is constructed using the equivalent integral form which can be found after some transformations as a Volterra integral equation. It relates the unknown time functions $f(t)=S_{n m}^{c, s}\left(R_{c}, t\right)$ to the surface data $\Phi(t)=\Phi_{n m}^{c, s}(t)=S_{n m}^{c, s}\left(R_{\sigma}, t\right)$ by

$$
\Phi(t)=A f(t)=\int_{0}^{t} k(t-\tau) f(\tau) \mathrm{d} \tau,
$$

where the kernel $k$ contains all the dependencies on $r, R_{\sigma}, R_{c}, n, m$, and $\sigma_{M}(r)$. As the kernel in general is not available analytically, an approximating matrix $\left(a_{i k}\right)$ is determined, which is related to systems of base functions that decompose the time functions $f(t)$ and $\Phi(t)$, respectively. Thus, the solution of the downward continuation problem comprises two steps:

First, the determination of this matrix requires the solution of stable boundary value problems (upward continuations): the unknown function $f(t)$ can be presented by the basis functions $\left\{e_{k}(t)\right\}, f=\sum f_{k} e_{k}$. The upward continuation means the mapping $A\left(e_{k}(t)\right)$. These calculated functions have to be decomposed by a further series of arbitrary basis functions, $\left\{\chi_{i}\right\}$,

$$
\sum_{i} a_{i k} \chi_{i}(t)=A\left(e_{k}(t)\right)
$$

finally finding the matrix elements.

Second, the expansion coefficients, $\left\{f_{k}\right\}$, of the unknown function $f(t)$ remain as the proper unknowns of the downward continuation problem that is now given in discrete form as

$$
\Phi_{i}=\sum_{k} a_{i k} f_{k}, \quad \Phi=\sum_{i} \Phi_{i} \chi_{i}
$$


i.e. with $\Phi_{i}$ as coefficients of the development of the geomagnetic boundary values. The unknown coefficients $\left\{f_{k}\right\}$ are determined by a regularization of the type

$$
\min \left\|\left(f_{k}\right)\right\|_{\beta} \quad \text { subject to }\left\|\left(a_{i k}\right)\left(f_{k}\right)-\left(\Phi_{i}\right)\right\|_{\alpha} \leq \epsilon,
$$

which accounts especially for the error $\epsilon$ of the data $(\Phi)$ at $R_{\sigma}$ while the first term searches for optimal smoothness of the solution at $R_{c}$ according to the applied norm $\|\cdot\|_{\beta}$, here the $L_{2}$ norm.

\section{THE POLOIDAL EM COUPLING TORQUE}

The EM coupling torque on the mantle is defined in Section 2.1 by eq. (1). Using the Maxwell equation curl $\mathbf{B}=\mu_{0} \mathbf{j}$, we can completely express the torque by the magnetic flux,

$$
\mathbf{L}=\mu_{0}^{-1} \int_{V_{M}} \mathbf{r} \times(\operatorname{curl} \mathbf{B} \times \mathbf{B}) \mathrm{d} V .
$$

Rochester (1962) has shown, that the volume integral in eq. (18) can be transformed into a surface integral over the CMB (surface $F_{c}$, radius $R_{c}$ ),

$$
\mathbf{L}=\mu_{0}^{-1} \int_{F_{c}}\left[(\mathbf{r} \times \mathbf{B})(\mathbf{B} \cdot \mathbf{n})-\frac{\left(B^{2}\right)}{2}(\mathbf{r} \times \mathbf{n})\right] \mathrm{d} F .
$$

In eq. (19), $\mathbf{n}$ is the surface unit vector pointing out of the volume $V_{M}$, i.e. towards the core. Conventionally, it is assumed that the departure of $F_{c}$ from a sphere is so small that $\mathbf{n}=-\mathbf{e}_{r}$ approximately holds, i.e.

$$
\mathbf{L}=-\mu_{0}^{-1} \int_{F_{c}}(\mathbf{r} \times \mathbf{B}) B_{r} \mathrm{~d} F,
$$

where $B_{r}$ is the $r$ component of $\mathbf{B}$. Eq. (20) is exact on a sphere and a good approximation on a surface with small ellipticity. The $z$ component of $\mathbf{L}$, which is the subject of this paper, is then given by

$$
L_{z}=-\mu_{0}^{-1} \int_{F_{c}} r B_{r} B_{\varphi} \sin \vartheta \mathrm{d} F,
$$

where $r, \vartheta, \varphi$ are the spherical coordinates.

As shown in the Appendix, $B_{r}$ is completely represented by the poloidal part of the geomagnetic field. Dividing $\mathbf{B}$ in eq. (20) (or $B_{\varphi}$ in eq. (21)) in its poloidal and toroidal parts according to eq. (3), respectively, we obtain two parts of the torque, one of which is generated by poloidal field components only, and the other by a mixture of both field parts. Following Stix and Roberts (1984), we denote them as poloidal and toroidal torque, respectively.

The poloidal torque can completely be inferred from the observed part of the geomagnetic field, whereas the toroidal torque is highly dependent of assumptions and results of core-flow models. 
By eqs. (26) and (28) (see Appendix) the poloidal part of $L_{z}$ in eq. (21), $L_{p z}$, can then be written as

$$
L_{p z}=+\mu_{0}^{-1} \int_{F_{c}} \frac{\Omega S}{r} \frac{\partial \tilde{S}}{\partial \varphi} \mathrm{d} F,
$$

where $\Omega$ is given by eq. (10) and $\tilde{S}$ is defined in eq. (4).

Using $\mathrm{d} F=R_{c}^{2} \sin \vartheta \mathrm{d} \vartheta \mathrm{d} \varphi$, the normalization condition (9) for $P_{n m}$ and eq. (11), we obtain as final expression for $L_{p z}$

$$
L_{p z}=\frac{2 \pi R_{c}^{2}}{\mu_{0}} \sum_{n=1}^{n_{\max }} \sum_{m=1}^{n} \frac{m n(n+1)}{2 n+1} \frac{(n+m) !}{(n-m) !}\left[S_{n m}^{s} \frac{\partial S_{n m}^{c}}{\partial r}-S_{n m}^{c} \frac{\partial S_{n m}^{s}}{\partial r}\right]_{R_{c}}
$$

with the spherical harmonic coefficients defined in eq. (8), and determined by NHDC at the CMB

\section{MODEL ASSUMPTIONS AND DATA}

\subsection{Conductivity models}

The origin of a lower-mantle conductivity of sufficient magnitude has not been consistently explained up to now. Shankland et al. (1993) inferred from laboratory experiments that $\sigma_{M}$ increases exponentially to the CMB and reaches a value of $10 \mathrm{Sm}^{-1}$, which is too low to produce significant EM torques. Holme (1998b) found that a conductance of the order of magnitude of $10^{8} \mathrm{~S}$ of the lowermost shell of the mantle is necessary to produce the observed $\Delta$ LOD by EM coupling. Buffett (1992) concluded that EM core-mantle coupling is required for the retrograde annual nutation of the Earth to be out of phase and estimated a shell of $0.2 \mathrm{~km}$ at the bottom of the mantle with $\sigma_{M}=5 \cdot 10^{5} \mathrm{~S} \mathrm{~m}^{-1}$ (a shell of $1 \mathrm{~km}$ with $10^{5} \mathrm{~S} \mathrm{~m}^{-1}$ would have the same conductance). New laboratory experiments show that such high conductivity values are possible at the bottom of the mantle (e.g. Dubrovinsky et al., 2003). However, other mechanisms could also give such values, including Buffett's et al. (2000) sedimentation in the core mechanism, and most recently the possibility that the post-perovsike phase might have an elevated electrical conductivity (e. g. Ono et al., 2006).

For our investigations we assume a only radially dependent parametrization of the conductivity, $\sigma_{M}$, which approximates these findings. We know about the limitations due to this assumption. However, we focus here on decadal variation of LOD, which is less affected by the assumption of lateral homogeneous conductivity than the long-term trend (Holme, 2000). In addition, also the NHDC is based upon this assumption. Therefore, we vary $\sigma_{M}$, radial dependent only, in a certain range, to check the dependence of $L_{p z}$ on the conductivity. The basic geometric set up is shown in fig. (1) and the four considered conductivity models 


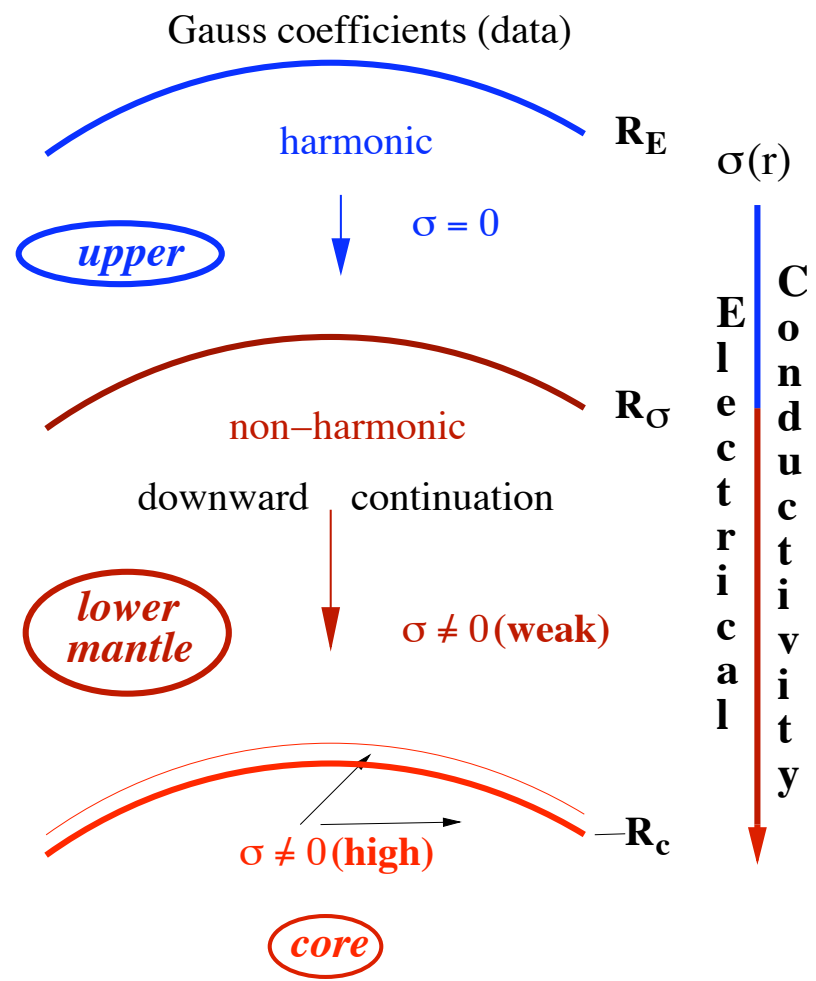

Figure 1: The shell model of the Earth's used for the non-harmonic downward continuation, with $R_{E}=6371 \mathrm{~km}, R_{\sigma}=5480 \mathrm{~km}$ and $R_{c}=3485 \mathrm{~km}$.

of the lowermost shells are given by

$$
\text { model } k \quad \sigma_{M}(r)= \begin{cases}0 & r>R_{\sigma} \\ 10 \cdot\left(\frac{R_{c}}{r}\right)^{5} \Omega^{-1} \mathrm{~m}^{-1} & R_{c}+x_{k}<r \leq R_{\sigma} \\ 1 \cdot 10^{5} & R_{c} \leq r \leq R_{c}+x_{k}\end{cases}
$$

$$
\text { with } \begin{aligned}
k & =1,2,3,4 \\
x_{1} & =2.0 \mathrm{~km}, x_{2}=1.5 \mathrm{~km}, x_{3}=1.0 \mathrm{~km}, x_{4}=0.5 \mathrm{~km} \\
R_{c} & =3485 \mathrm{~km} \\
R_{\sigma} & =5480 \mathrm{~km}
\end{aligned}
$$

where the radii are displayed in fig. (1). The power function is according to Shankland's et al. (1993) results, and $k$ is the counter for the number of the model used.

\subsection{Satellite supported geomagnetic field models}

The data used in this study are monthly spaced sequences of $\mathrm{SH}$ expansions of the geomagnetic field at the Earth surface developed by Wardinski and Holme (2006), $\mathrm{C}^{3} \mathrm{FM}$ 
$\left(n_{\max }=15\right)$, and Sabaka et al. (2004), CM4 $\left(n_{\max }=13\right)$, between 1980 and 2000. Both field models are based on different modelling approaches. The CM4 model uses a comprehensive approach (e.g. Sabaka et al.,2002;2004), where the magnetic field is parametrized using satellite and observational data for separate spheres, i.e. crust, ionosphere and the internal (residual) field. In contrast, Wardinski and Holme (2006) apply a hybrid approach using satellite field models for the epochs (Magsat and Ørsted) to constrain a field model derived from ground-based data for the time interval $1980-2000$.

Figure 2 (a) and (b) show for the geomagnetic field model $\mathrm{C}^{3} \mathrm{FM} B_{r}$ and $B_{\phi}$ for the year 1995 at the CMB for $n_{\max }=8$, respectively. For the comparison with the field model CM4 in sub-figure (c) the related difference $\Delta B_{r}$ is presented, which is in the order of $1 \%$ of $B_{r}$ at the CMB. Beside the significant extrema with a dipole structure in South America and the Indian Ocean (around $\pm 7000 \mathrm{nT}$ ) differences appear with a spatial structure, which seems to be related with the high degree and order pattern of the $\mathrm{SH}$ degree $n=8$. The corresponding difference $\Delta B_{\phi}$ between both field models is shown in the sub-figure (d). The most outstanding difference $(\sim-7000 \mathrm{nT})$ is located in south Atlantic, close to the South American coast, which is about $2 \%$ of $B_{\phi}$ at the CMB. Moreover, a similar spatial structure (stripe pattern) like that in sub-figure (c) appear, which is not so strongly pronounced. In (e) and (f) the differences $\Delta B_{r}$ and $\Delta B_{\phi}$ are shown for the full set of spectral coefficients for each of the models. The spatial pattern of the differences is similar to that of the considered maximum spectral degree of $n_{\max }=15$ and $n_{\max }=13$, respectively. In sub-figure (e) the differences are in the order of $15 \%$ of $B_{r}$ at the CMB, whereas in sub-figure (f) values of $25 \%$ of $B_{\phi}$ can be obtained. In contrast to the comparison for $n_{\max }=8$ the results in subfigure (e) and (f) seem to be artificial and do not reflect different spatial information of the geomagnetic models.

For a further understanding of the differences fig. 3 shows the power-spectrum (LowesMauersberger spectrum) of the secular variation of the model CM4 and $\mathrm{C}^{3} \mathrm{FM}$. From degree $n=5$ onwards the spectra differ, but initially in small range. For higher degrees the power is still increasing for CM4 whereas for $\mathrm{C}^{3} \mathrm{FM}$ the influence of the damping is significant and the power in the degrees $n=12$ to $n=15$ is reduced by nearly one order of magnitude. This is mainly a result of the different regularization for both geomagnetic field models. The secular variation is used for the regularization of $\mathrm{C}^{3} \mathrm{FM}$ at the $\mathrm{CMB}$, but for $\mathrm{CM} 4$ at the earth's surface.

Moreover, fig. 4 shows the power-spectra of both field models and of the differences in the Gauss coefficients. Up to degree $n=9$ both spectra of the field models have similar power and the difference spectrum is more than five orders of magnitude smaller. From degree $n=10$ onwards the power-spectrum of CM4 increases again, whereas the values for $\mathrm{C}^{3} \mathrm{FM}$ decrease to degree $n=15$ by more than one order of magnitude. The related power-spectrum of the differences in the Gauss coefficients shows a significant increase from degree $n=10$ to $n=13$ from $10^{5} \mathrm{nT}^{2}$ to $3 \cdot 10^{7} \mathrm{nT}^{2}$.

This indicates the differences in the regularization of both geomagnetic field models and the uncertainties of both models in resolving the field on $\mathrm{SH}$ degrees larger then $n=9$ to $n=10$. Therefore, we restrict our interpretation of the resulting torques to the maximum $\mathrm{SH}$ degree of $n_{\max }=8$. 


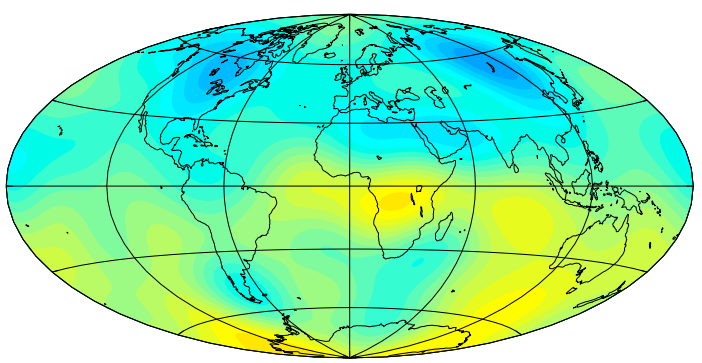

[1000nT]

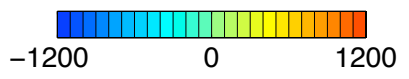

(a) $B_{r}\left(r=R_{c}\right)$ for $n_{\max }=8$

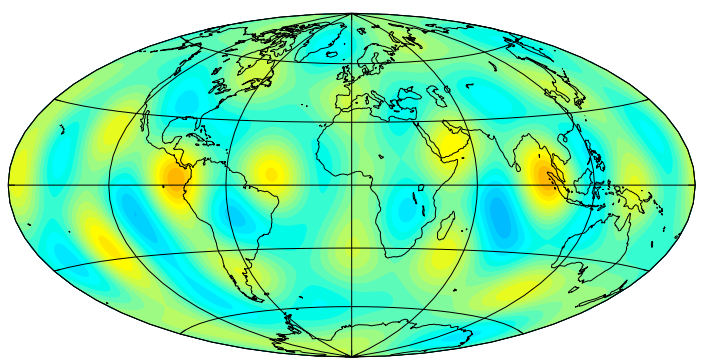

[1000nT]

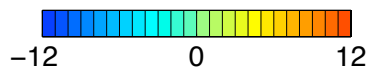

(c) $\Delta B_{r}\left(r=R_{c}\right)$ for $n_{\max }=8$

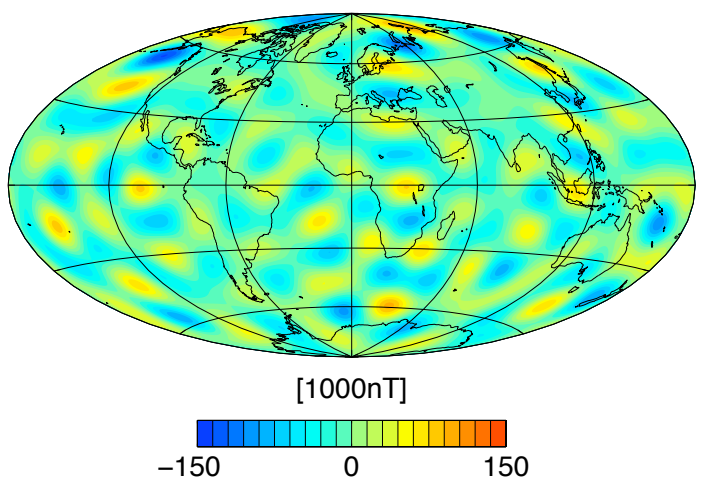

(e) $\Delta B_{r}\left(r=R_{c}\right)$

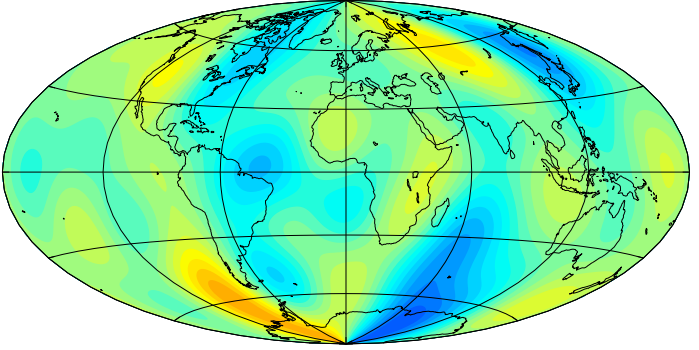

[1000nT]

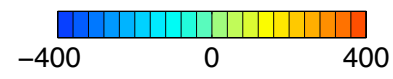

(b) $B_{\phi}\left(r=R_{c}\right)$ for $n_{\max }=8$

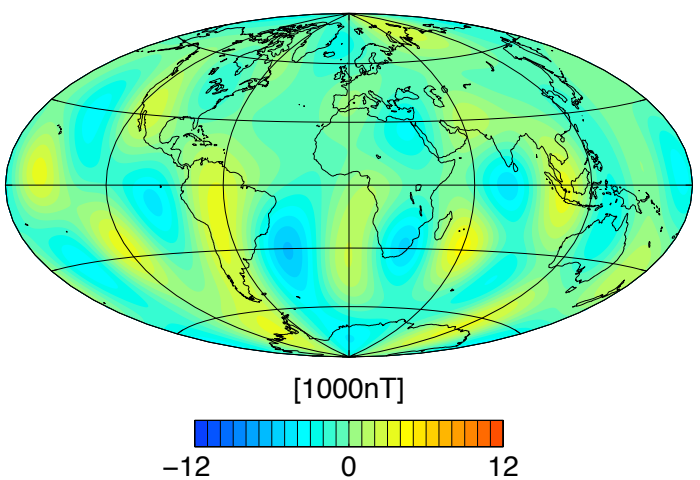

(d) $\Delta B_{\phi}\left(r=R_{c}\right)$ for $n_{\max }=8$

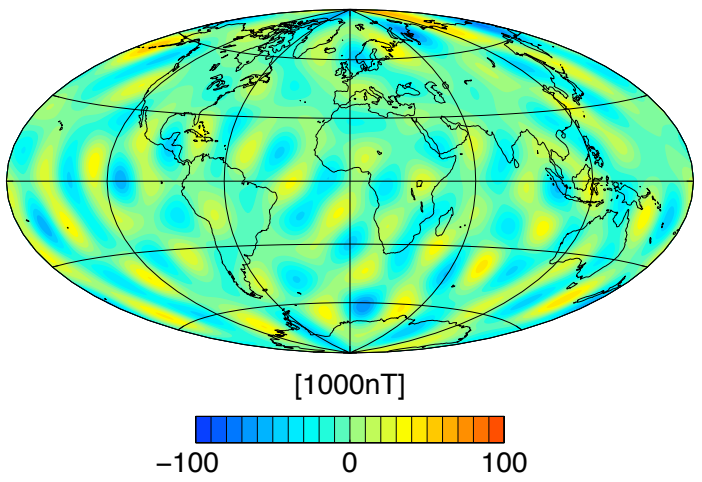

(f) $\Delta B_{\phi}\left(r=R_{c}\right)$

Figure 2: Comparison of the magnetic field models for the year 1995 at the CMB $\left(r=R_{c}\right)$. In (a) and (b) are shown $B_{r}$ and $B_{\phi}$, respectively, for $n_{\max }=8$ using the field model $\mathrm{C}^{3} \mathrm{FM}$. The related differences between this components and thus of the field model CM4 are shown in (c) and (d), respectively. Moreover, in (e) and (f) this differences are shown for the same field models but using now $n_{\max }=15$ for $\mathrm{C}^{3} \mathrm{FM}$ and $n_{\max }=13$ for CM4, respectively.

\subsection{LOD data and mechanical torques}

The data of $\triangle \mathrm{LOD}$ and the atmospheric angular momentum (AAM) are obtained from IERS (C04 and AAM NCEP-NCAR reanalysis time series). We subtract the LOD-equivalent of AAM, obtaining residuals $\triangle \mathrm{LOD}_{o b s-A A M}$ to be interpreted by core-mantle coupling, i.e. we 


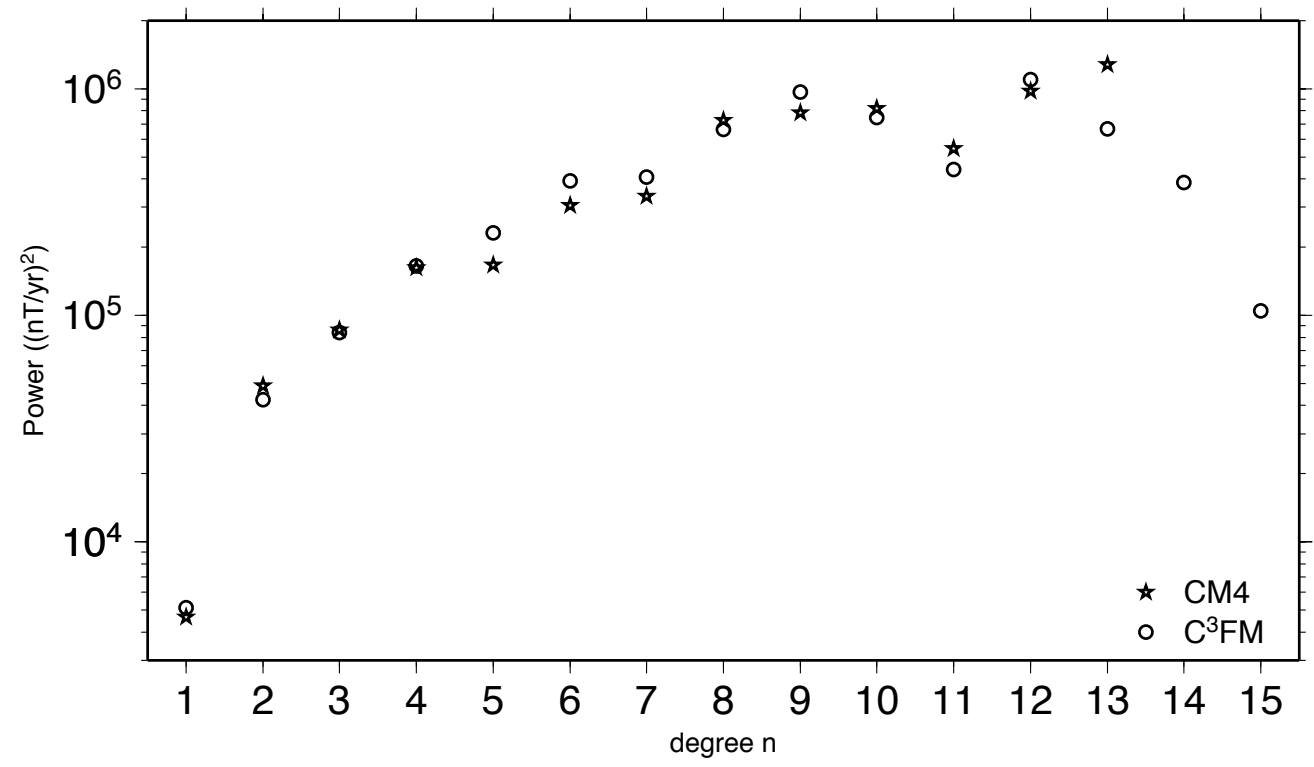

Figure 3: Degree-power spectra (Lowes-Mauersberger) of the secular variation of the $\mathrm{C}^{3} \mathrm{FM}$ and the CM4 model at the CMB by NHDC.

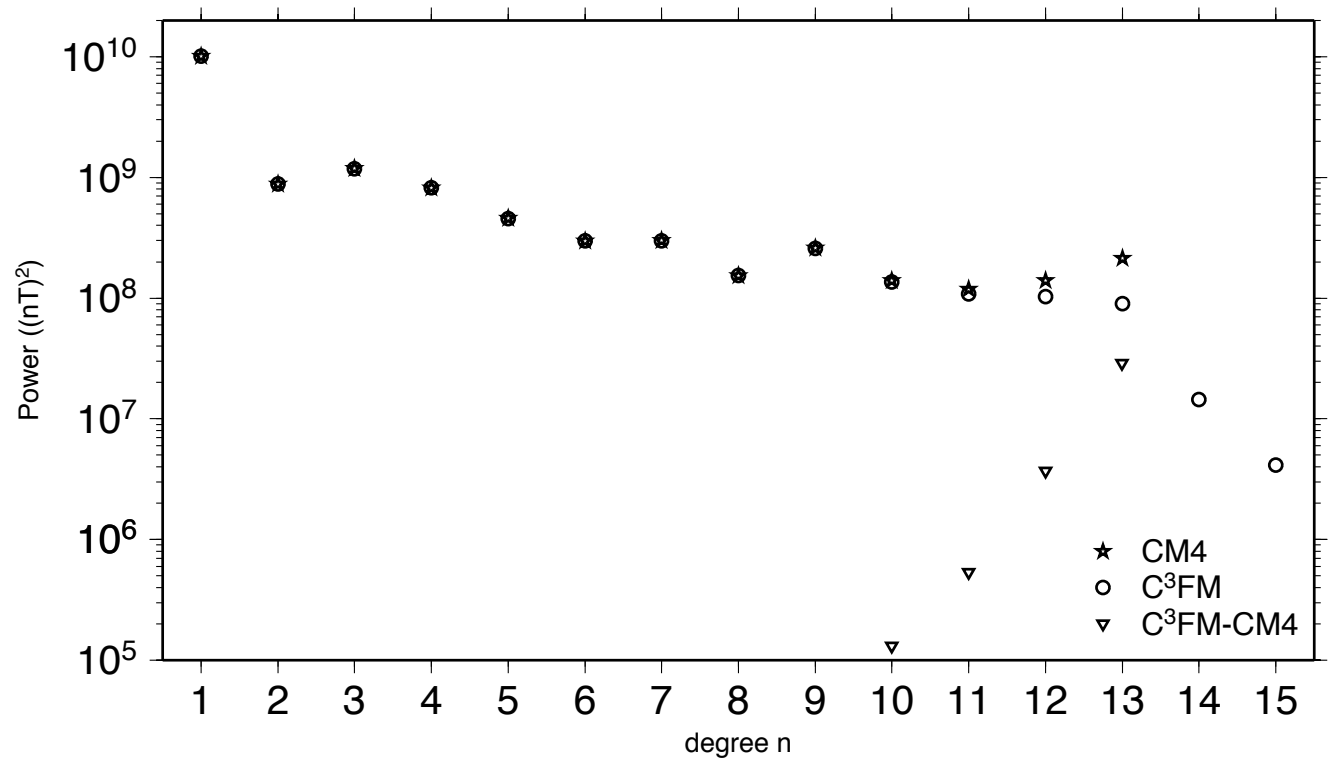

Figure 4: Degree-power spectra of the geomagnetic field models $\mathrm{C}^{3} \mathrm{FM}$ and the CM4 at the CMB by NHDC. In addition is shown the spectrum of the differences in the Gauss coefficients, where the values are smaller than $10^{5} \mathrm{nT}^{2}$ for degrees smaller than 10 .

imply that the contributions of ocean and the continental hydrosphere are insignificant for our investigations. At the latest, if we will switch over to a finer and more comprehensive investigation of a joint coupling in future, we must consider these contributions too. The values of $\triangle \mathrm{LOD}_{\text {obs }-A A M}$ are shown in fig. 5 .

To derive the necessary mechanical torque, $L_{z}^{\text {mech }}$, from $\triangle \mathrm{LOD}_{\text {obs-AAM }}$ we smooth the time series by a standard running mean procedure, and determine $L_{z}^{\text {mech }}$ according to the 


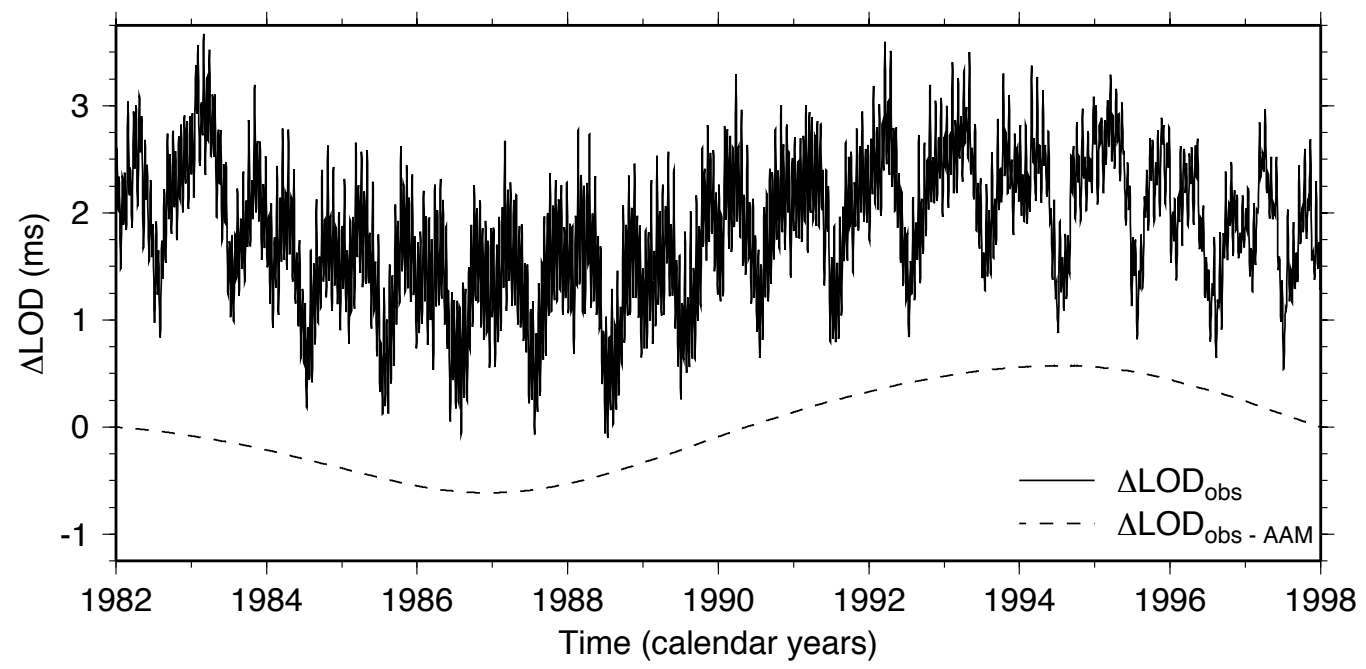

Figure 5: Smoothed LOD variations, $\triangle \mathrm{LOD}_{\text {obs }-A A M}$, obtained after subtraction of $\triangle \mathrm{LOD}$ excited by AAM from their observed values and de-trended.

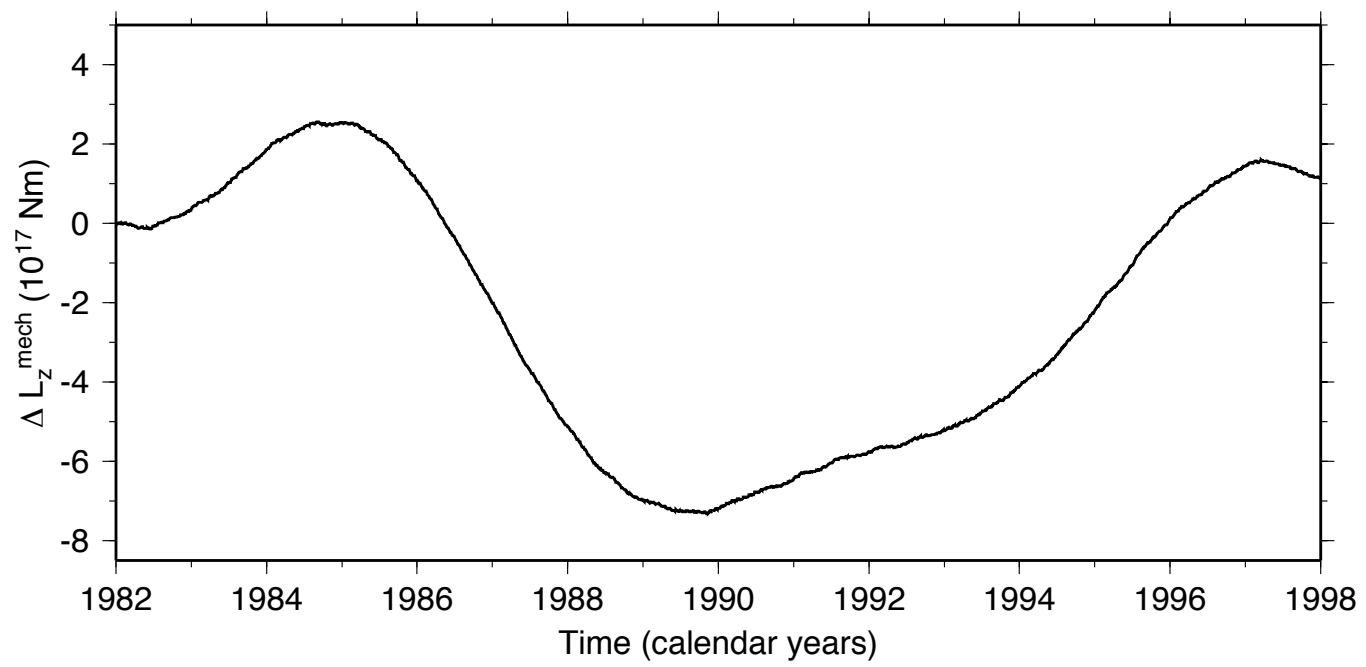

Figure 6: Variations of $L_{z}^{\text {mech }}$ necessary to produce $\Delta \mathrm{LOD}_{o b s-A A M}$. They are obtained by numerical differentiation according to eq. (25).

third component of the Euler-Liouville equation for the angular velocity, $\omega_{M}$, of the mantle

$$
C_{M} \frac{\mathrm{d} \omega_{\mathrm{M}}}{\mathrm{d} t}=-\frac{2 \pi}{T_{0}^{2}} \frac{\mathrm{d} \Delta \mathrm{LOD}}{\mathrm{d} t}=L_{z}^{m e c h}
$$

where $T_{0}=86400 \mathrm{~s}$ is the length of day, and $\Delta \mathrm{LOD}$ is the observed variation of $T_{0}$. The time derivative of $\triangle \mathrm{LOD}$ in eq. (25) will be computed by B-spline interpolation and the related analytical derivation using cubic B-splines. The values of $L_{z}^{m e c h}$ are shown in fig. 6 . 


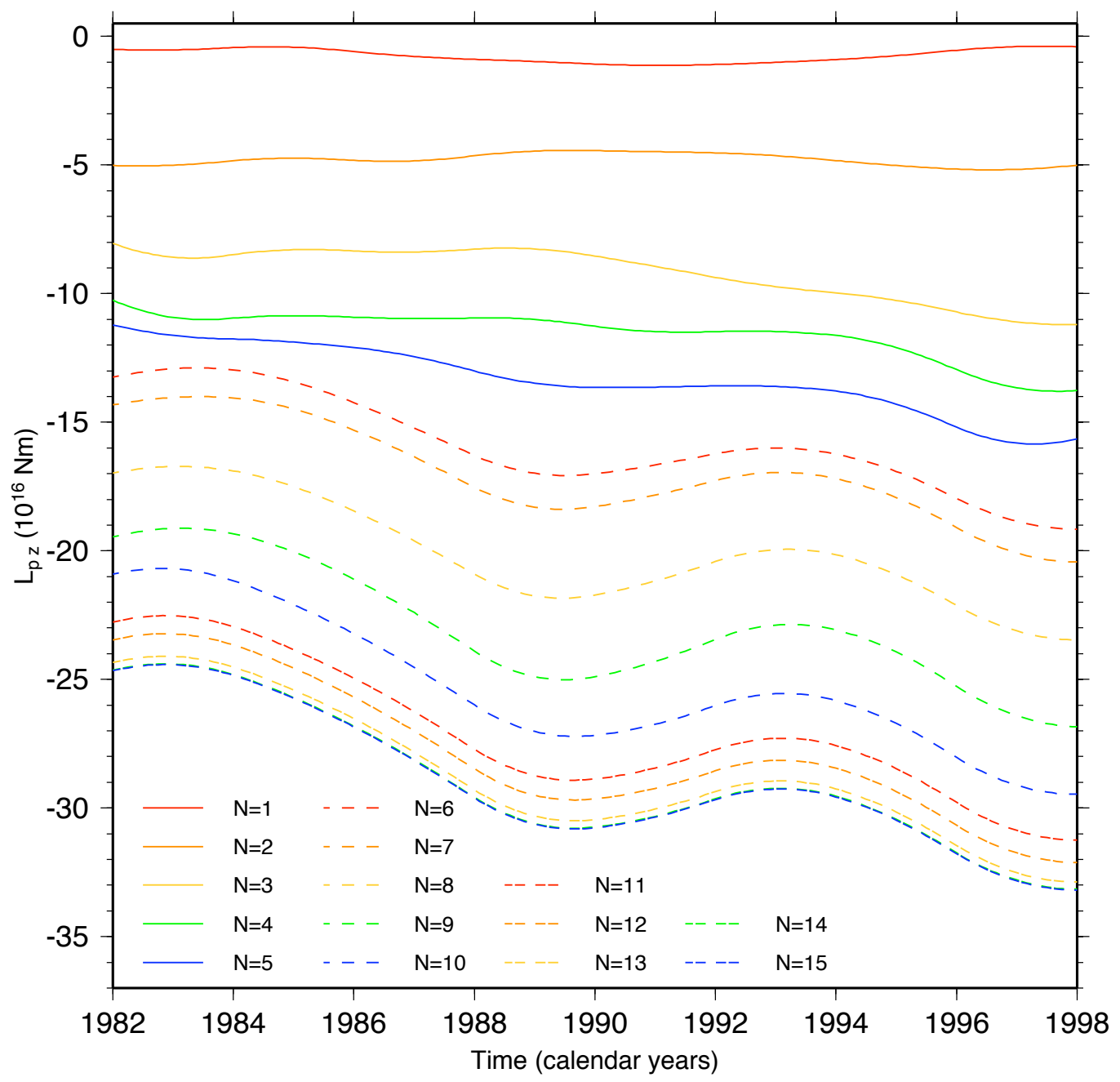

Figure 7: $L_{p z}$ in dependence on the cumulative degree $\mathrm{N}$ and time for the conductivity model 1 (see eq. (24)) and the geomagnetic field model $\mathrm{C}^{3} \mathrm{FM}$.

\section{RESULTS}

We will first study the dependence of $L_{p z}$ and its time behavior on the SH degree $n$ of the geomagnetic field models. Those are developed for the maximum degree, $n_{\max }=13$ or $n_{\max }=15$, respectively (Section 4 ), giving a set of Gauss coefficients for $n=1,2,3, \ldots, n_{\max }$ and $m=0, \ldots, n$. The EM torques are computed in dependence on the cumulative degree $N$ according to eq. (23) for $n=1,2,3, \ldots, N$, i.e. $L_{p z}(N)$ contains the contributions of the modes with lower $n$ too. The absolute values of the EM torque $L_{p z}$ are shown in figs. 7 and 8. For the model $\mathrm{C}^{3} \mathrm{FM}$ the time behavior of $L_{p z}$ changes significantly up to $N=6$. In contrast, for the higher degrees $(N=7, \ldots, 12)$ the resulting EM torque is nearly shifted without any pronounced change in the time behavior and for the degrees $N=13, \ldots, 15$ the torque and its time behavior vary only in a small range of around $2 \%$. This observations can 


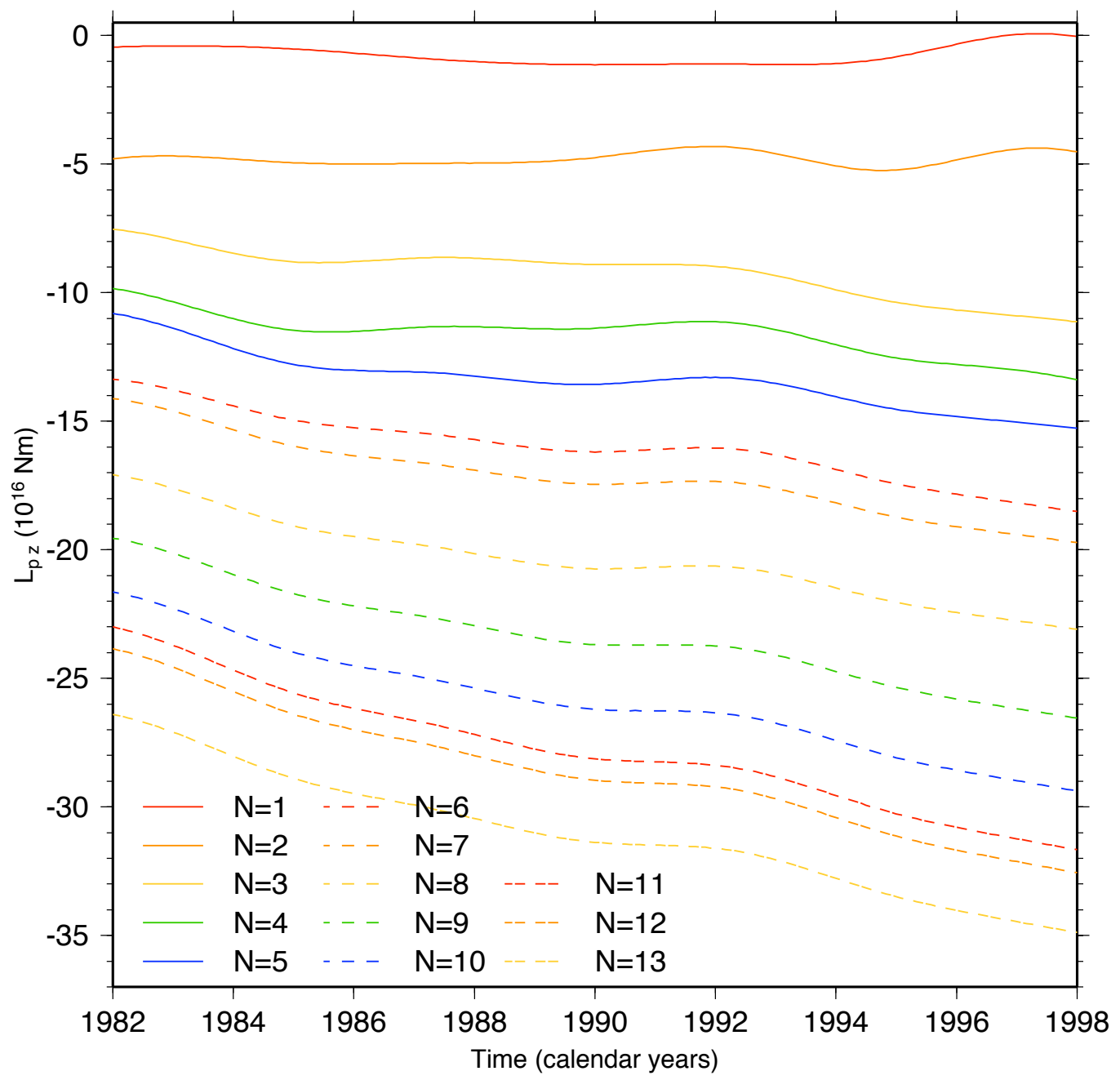

Figure 8: $L_{p z}$ in dependence on the cumulative degree $\mathrm{N}$ and time for the conductivity model 1 (see eq. (24)) and the geomagnetic field model CM4.

be partly explained by the uncertainties of the field model and its secular variation at SH degrees $n=9$ and above (c. f. discussion of fig. 4 and 3). The observed shift with decreasing offsets in $L_{p z}(N)$ results most likely from the unreliability of the coefficients for $N>10$.

The changes in the time behavior in $L_{p z}(N)$ for the model CM4 are significant up to the cumulative degree $N=6$ (fig. 8). For all higher degrees the change in the EM torque can be approximated by a shift and a small variation in a linear trend. Also here the related powerspectra of the geomagnetic field CM4 (fig. 4) and the related secular variation (fig. 3) give additional insight in this behavior. For CM4 both spectra increase for the higher degrees, which is clearly visible in the change of $L_{p z}(N)$ even for the highest cumulative degrees $N=11, \ldots, 13$. This observations also confirm the restriction of $L_{p z}(N)$ to the cumulative degree $N=8$.

Figures 9 and 10 show the variations, $\Delta L_{p z}(N)$, of $L_{p z}(N)$ with respect to their linear trends for the cumulative degree $N$ and both geomagnetic field models. Following the argumentation above, we restrict our discussion here to $\Delta L_{p z}(N)$ for $N \leq 8$, which allows us in this case to conclude, that the differences in figs. 9 and 10 are related to the chosen geomagnetic field model. The curves of $\Delta L_{p z}(N)$ computed from $\mathrm{C}^{3} \mathrm{FM}$ and $\mathrm{CM} 4$ are rather 


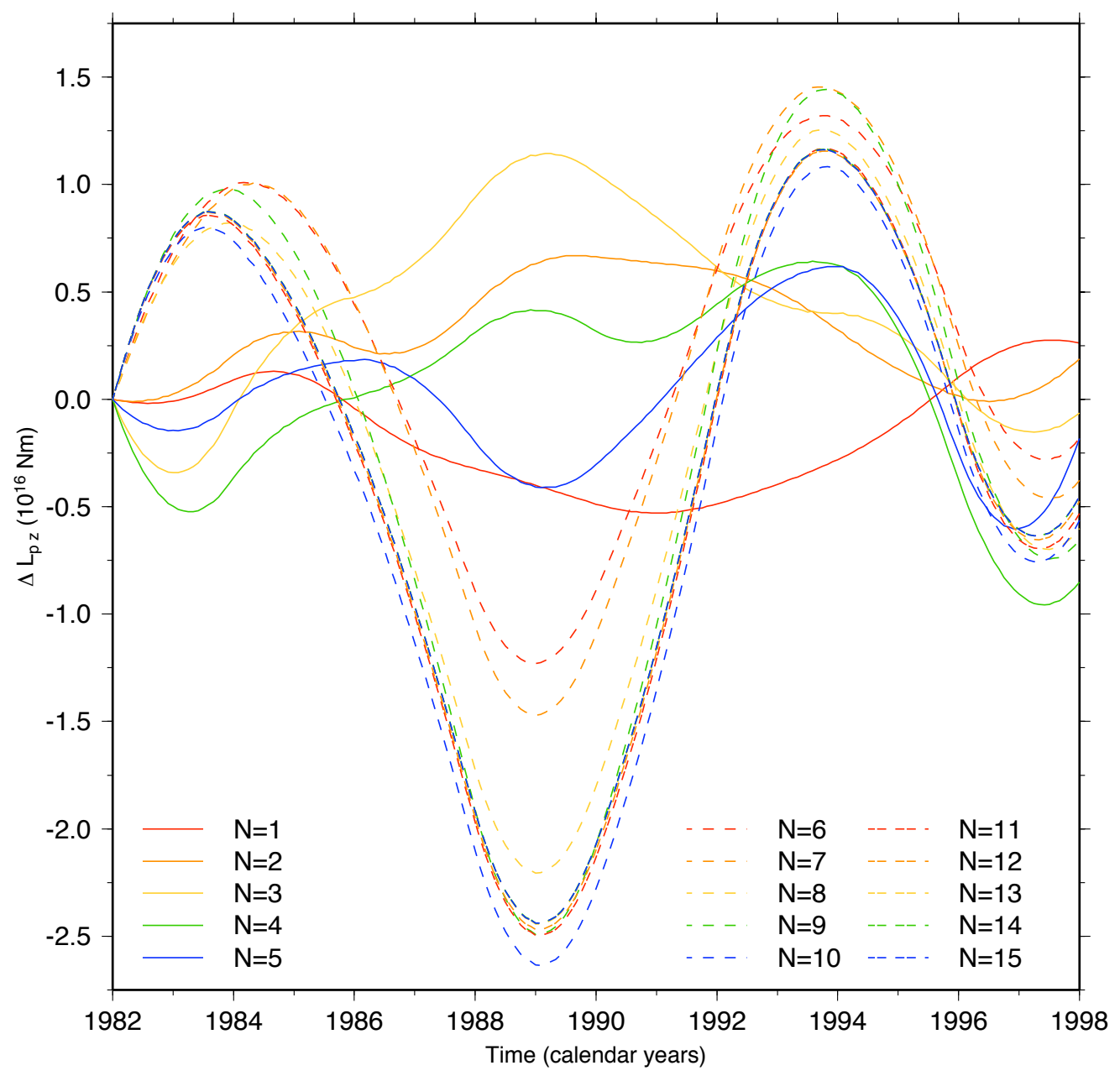

Figure 9: The variations, $\Delta L_{p z}$, with respect to their linear trends in dependence on the cumulative degree $\mathrm{N}$ for the conductivity model 1 (see eq. (24)) and the geomagnetic field model $\mathrm{C}^{3} \mathrm{FM}$.

incompatible. This is the second result, we want to emphasize. Again, this can be explained by the different modelling approaches to set up the field models.

Finally, we will show the dependence on $\sigma_{M}$ and compare $\Delta L_{p z}$ with the mechanical torque. Figures 11 and 12 show the dependence of $\Delta L_{p z}$ on the conductivity model for $n_{\max }=8$ and the comparison between $\Delta L_{p z}$ and $\Delta L_{z}^{\text {mech }}$ for the $\sigma_{M}$-model 1 , respectively. The curves in fig. 11 show an expected linear influence of $\sigma_{M}$, i.e. an increase in $\sigma_{M}$ does not change the temporal structure of the torque. A thickening of the highly conductive layer above the CMB from $0.5 \mathrm{~km}$ to $2.0 \mathrm{~km}$ results roughly in a four times larger EM torque. For comparison, $\Delta L_{p z}$ is also plotted in fig. 11, which is computed from CM4 for the conductivity model 1. Beside a smaller amplitude we find a significantly different time behavior.

In addition, fig. 11 shows significant differences in $\Delta L_{p z}$ between both geomagnetic field models used as input data, demonstrated for the conductivity model 1. Figure 12 shows the poloidal axial EM torque and the axial torque, $\Delta L_{z}^{m e c h}$, computed from the observed values of $\Delta \mathrm{LOD}$, whereas $\Delta L_{p z}$ is in the order of $10^{16} \mathrm{Nm}$ and $\Delta L_{z}^{\text {mech }}$ is in the order of $10^{17} \mathrm{Nm}$. Therefore, the plotted difference between both axial torques is nearly identically 


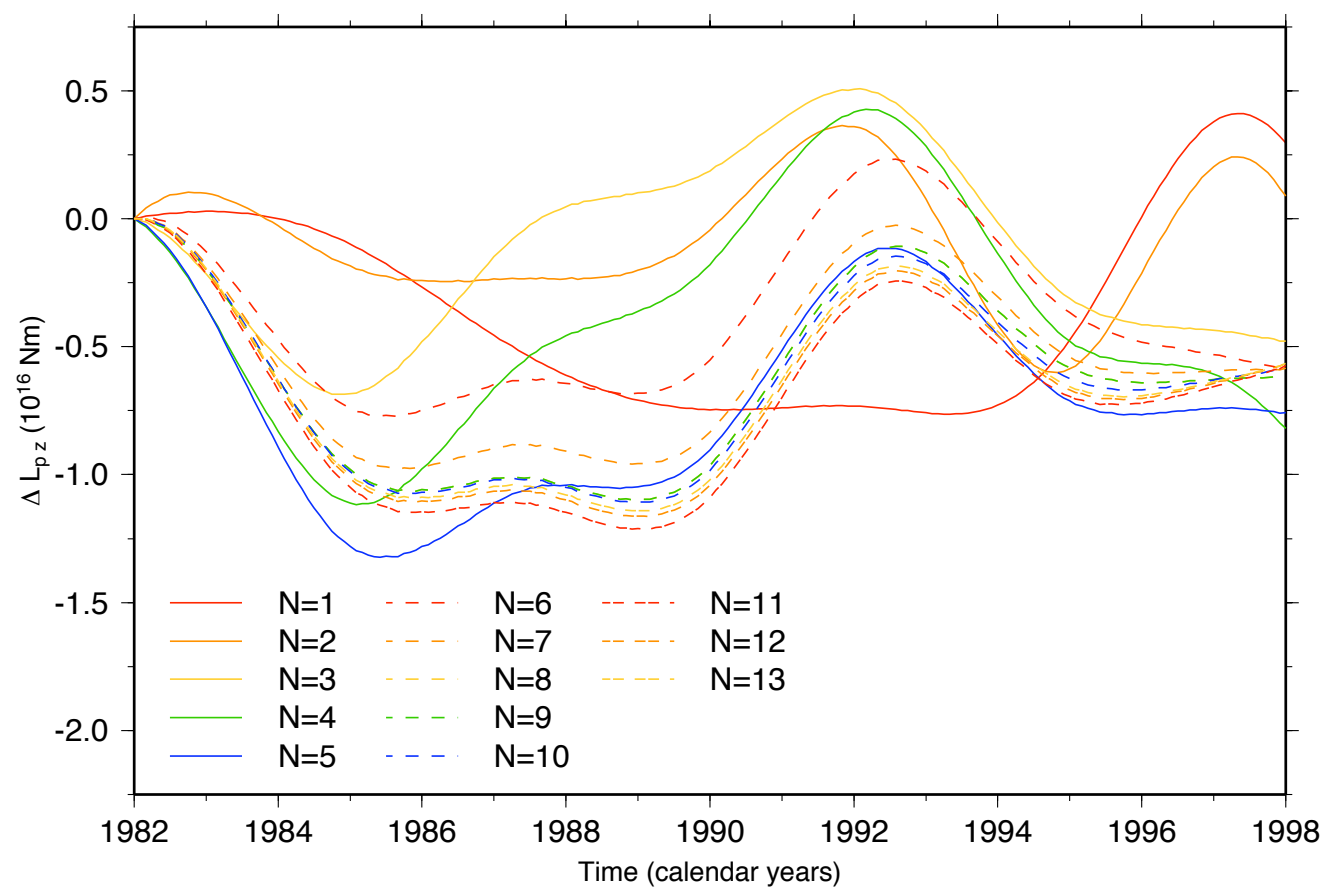

Figure 10: The variations, $\Delta L_{p z}$, with respect to their linear trends in dependence on the cumulative degree $\mathrm{N}$ for the conductivity model 1 (see eq. (24)) and the geomagnetic field model CM4.

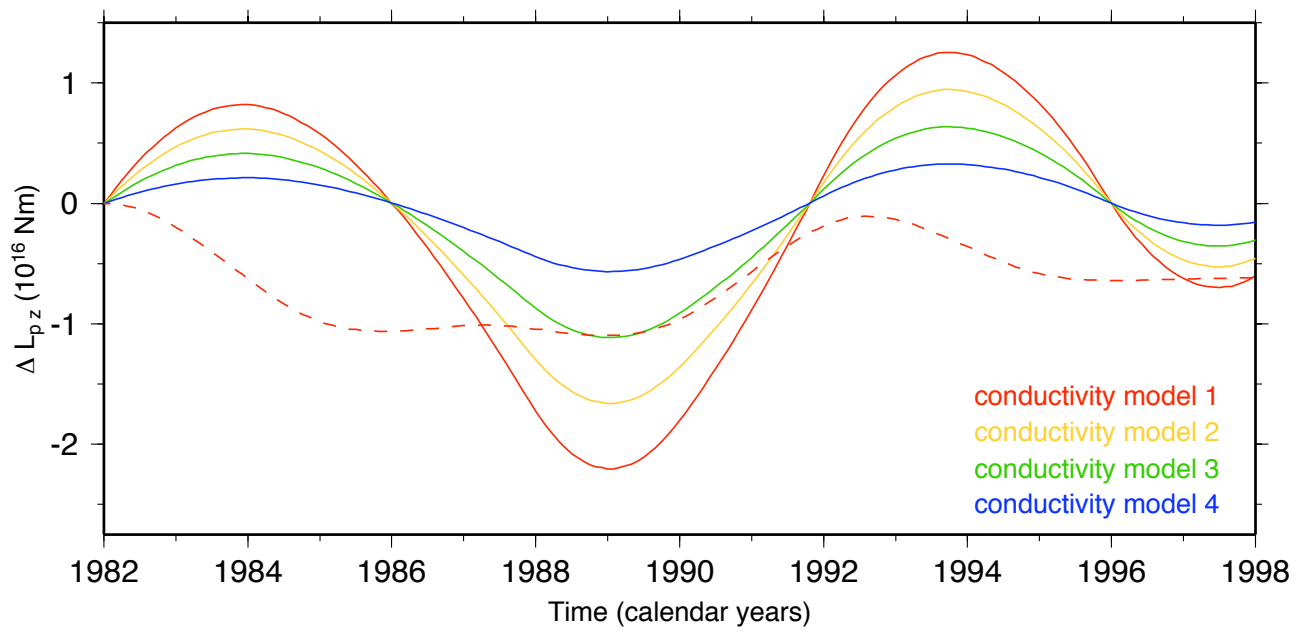

Figure 11: The dependence of $\Delta L_{p z}$ on the conductivity models (see eq. (24)) and time for $n_{\max }=8\left(\mathrm{C}^{3} \mathrm{FM}\right.$, solid lines; CM4, dashed line).

with $\Delta L_{z}^{\text {mech }}$. Apart from this observations, a time shift between the EM torque and the 'mechanical' torque is obtained, which is about $1.5 \mathrm{yr}$. This is in agreement with the conclusion of Stix and Roberts (1984), computing a time constant of $\sim 1.6 \mathrm{yr}$ for the Earth's mantle. 


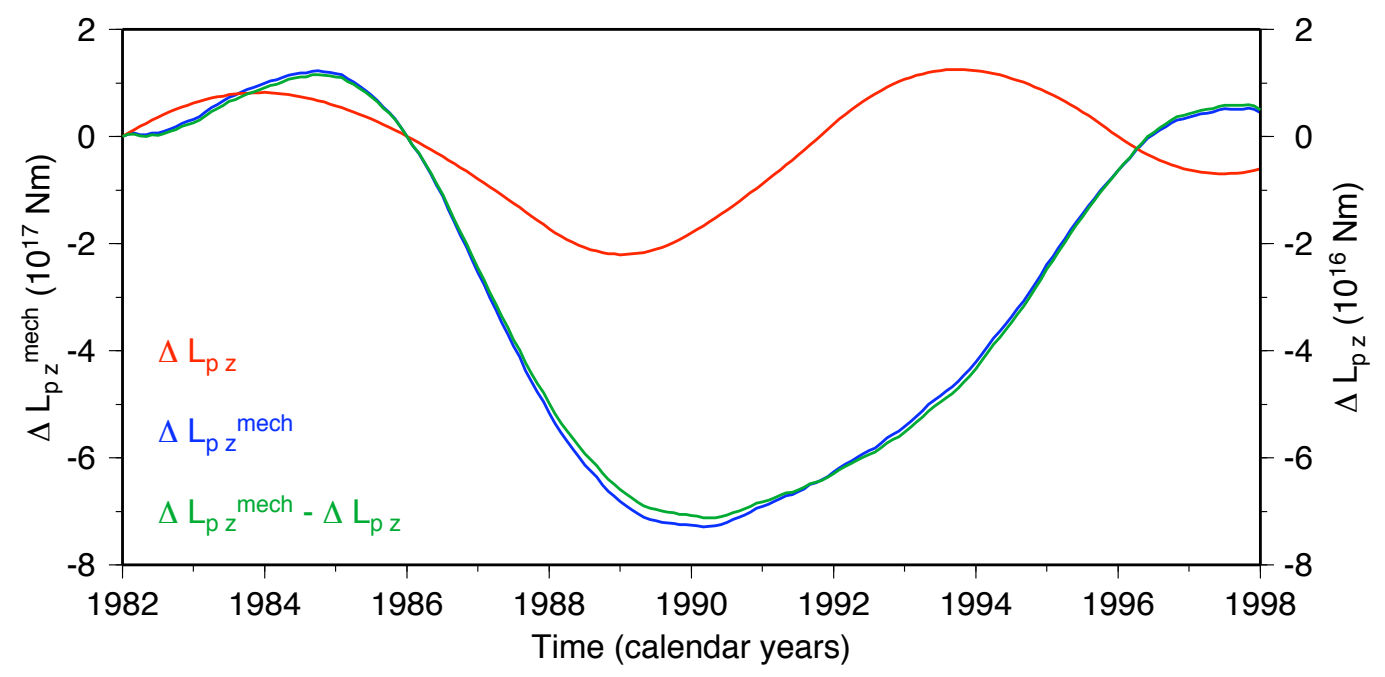

Figure 12: $\Delta L_{z}^{\text {mech }}$ and $\Delta L_{p z}$ for $n_{\max }=8\left(\mathrm{C}^{3} \mathrm{FM}\right.$, conductivity model 1, see eq. (24)) both centered about their mean value, and the difference between them.

\section{CONCLUSIONS}

The investigation of the dependence of $L_{p z}$ and $\Delta L_{p z}$ on the cumulative degree $N$ shows that we have to restrict the geomagnetic field models to $\mathrm{SH}$ expansions with maximum degree $n_{\max }=8$ for the computation of the torque variation. As discussed in Section 4.2, this is probably a consequence of an insufficient resolution of the geomagnetic field at higher degrees by the data and the algorithm.

Concerning the torque $L_{p z}$, the differences between the two geomagnetic field models used, particularly with respect to the temporal behavior, are not marginal even in the case for $n_{\max }=8$ (e.g. fig. 11). Our conclusions are: (i) the $\mathrm{SH}$ representation of the geomagnetic field models should be also consistent with observable physical quantities like LOD variations and (ii) we have to gain more insight into the sources for this (specially temporal) differences between the geomagnetic field models.

The comparison between EM and mechanical torques derived from $\triangle \mathrm{LOD}_{o b s-A A M}$ confirms what we know from earlier investigations: we cannot identify a best fitting conductivity model only by $\Delta L_{p z}$ and $\Delta L_{z}^{\text {mech }}$. The poloidal axial EM torque is more than one order of magnitude smaller than the mechanical torque. Therefore, we have to consider the toroidal EM torque, $L_{t z}$, which is a candidate for a further substantial contribution to the torque budget.

An identification of a best fitting conductivity model will only be possible within a comprehensive modelling of core-mantle coupling, where also additional coupling mechanisms are considered (e.g. Voorhies, 1991; Ponsar et al., 2003). Further investigations demand an improved reduction of the observed $\triangle \mathrm{LOD}$ by considering the influence of e.g. oceans and 
continental hydrosphere.

\section{ACKNOWLEDGMENTS}

The authors would like to thank R. Holme and J. Velímský for their constructive reviews. Most figures of this article were generated with a free software, GMT (Wessel and Smith, 1991). The work was partly funded by the research unit "Earth Rotation and Global Dynamic Processes (DFG FOR 584)" of the German Research Foundation.

\section{REFERENCES}

Ballani L., Greiner-Mai H. and Stromeyer D., 2002. Determining the magnetic field in the core-mantle-boundary zone by non-harmonic downward continuation. Geophys. J. Int., 149, $374-389$.

Benton E.R. and Whaler K.A., 1983. Rapid diffusion of the poloidal geomagnetic field through the weakly conducting mantle, a perturbation solution. Geophys. J. R. astr. Soc., 75, 77-100.

Buffett B.A., 1992. Constraints on magnetic energy and mantle conductivity from the forced nutations of the Earth. J. Geophys. Res., 97, 19,581-19,587.

Buffett B.A., Jeanloz R. and Garnero E., 2000. Sediments at the top of Earth's core. Science, 290, 5495-5497.

Dubrovinsky L., et al., (2003). Iron-silica interaction at extreme conditions and the electrically conducting layer at the base of the Earth's mantle. Nature, 422, 58-61.

Greiner-Mai H., 1987. The influence of the electromagnetic core-mantle coupling torques on Earth's rotation. Astron. Nachr., 308, 217-226.

Greiner-Mai H., 1993. Decade variations of the Earth's rotation and geomagnetic core-mantle coupling. J. Geomag. Geoelectr., 45, 1333-1345.

Greiner-Mai H., and Jochmann H., 1998. Correction to 'Climate variations and the Earth's rotation'. J. Geodyn., 25, 1-4.

Greiner-Mai H., Ballani L. and Stromeyer D., 2004. The poloidal geomagnetic field in a differentially rotating upper core layer. Geophys. J. Int., 158, 864-873.

Gubbins, D. and Roberts, P.H., 1987. Magnetohydrodynamics of the Earth's Core. In: Jacobs, J.A. (Ed.), Geomagnetism, Volume 2, Academic Press, London.

Holme R., 1998a. Electromagnetic core-mantle coupling - I. Explaining decadal changes in the length of day. Geophys. J. Int.,132, 167-180.

Holme R., 1998b. Electromagnetic core-mantle coupling - II. Probing deep mantle conductance. In: Gurnis M., Wysession M.E., Knittle E., Buffett B.A. (Eds.), The Core-Mantle Boundary Region. Geodynamics, 28, Copyright 1998 by the AGU, 139-151. 
Holme, R., 2000. Electromagnetic core mantle coupling III. Laterally varying mantle conductance. Phys. Earth Planet. Inter., 117, 329-344.

Krause, F. and Rädler, K.-H., 1980. Mean-Field Magnetohydrodynamics and Dynamo Theory. Akademie-Verlag, Berlin.

Olsen, N., Lühr, H., Sabaka, T. J., Mandea, M., Rother M., Tøffner-Clausen, L. and Choi, S., 2006. CHAOS - a model of the Earth's magnetic field derived from CHAMP, Ørsted, and SAC-C magnetic data. Geophys. J. Int., 166, 67-75.

Ono, S., Oganov, A. R., Koyama, T. and Shimizu, H., 2006. Stability and compressibility of the high-pressure phases of $\mathrm{Al}_{2} \mathrm{O}_{3}$ up to $200 \mathrm{GPa}$ : Implications for the electrical conductivity of the base of the lower mantle. Earth Planet. Sci. Lett., 246, 326-335.

Paulus, M. and Stix, M., 1989. Electromagnetic core-mantel coupling: The Fourier method for the solution of the induction equation. Geophys. Astrophys. Fluid Dynamics, 47, 237249 .

Ponsar, S., Dehant, V., Holme, R., Jault, D., Pais, A. and Van Hoolst, T., 2003. The Core and Fluctuations in the Earth's Rotation. In: Earth's Core: Dynamics, Structure, Rotation, eds. Dehant, V., Creager, K.C., Karato, S. and Zatman, S., AGU Geodynamic Series, 31, $251-261$.

Roberts, P. H., 1972. Electromagnetic Core-Mantle Coupling. J. Geomag. Geoelectr., 24, $231-259$.

Rochester M. G., 1960. Geomagnetic westward drift and irregularities in the Earth's rotation. Phil. Trans. R. Soc., London A252, 531-555.

Rochester M. G., 1962. Geomagnetic core-mantle coupling. J. Geophys. Res., 67, 48334836 .

Sabaka, T. J., Olsen, N., Langel, R. A., 2002. A comprehensive model of the quiet-time, near-Earth magnetic field: phase 3. Geophys. J. Int., 151, 32-68.

Sabaka, T. J., Olsen, N. and Langel, R. A., 2004. Extending comprehensive models of the Earth's magnetic field with Ørsted and CHAMP data. Geophys. J. Int., 159, 521-547.

Shankland T.J., Peyronneau J. and Poirier J.P., 1993. Electrical conductivity of the Earth's lower mantle. Nature, 366, 453-455.

Stix M., 1982. On electromagnetic core-mantle coupling. Geophys. Astrophys. Fluid Dynamics, 21, 303-313.

Stix M. and Roberts P.H., 1984. Time-dependent electromagnetic core-mantle coupling. Phys. Earth Planet. Inter., 36, 49-60.

Voorhies, C.V., 1991. Coupling an Inviscid Core to an Electrically Insulating Mantle. J. Geomag. Geoelectr., 43, 131-156.

Wardinski I. and Holme R., 2006. A time-dependent model of the earth's magnetic field and its secular variation for the period 1980 to 2000. J. Geophys. Res., 111, doi:10.1029/2006JB004401. 
Wessel, P., and Smith, W. H. F.,1991. Free software helps map and display data, Eos Trans. $A G U, \mathbf{7 2}, 445-446$.

\section{APPENDIX}

We will derive the boundary conditions as a consequence of the continuity of the components of $\mathbf{B}$, and show, how their mode form depends on the Gauss coefficients of the surface field. First, from eqs. (3)-(5) we can derive

$$
\begin{aligned}
& B_{r}=-\frac{1}{r}\left[\frac{1}{\sin \vartheta} \frac{\partial}{\partial \vartheta}\left(\sin \vartheta \frac{\partial S}{\partial \vartheta}\right)+\frac{1}{\sin ^{2} \vartheta} \frac{\partial^{2} S}{\partial \varphi^{2}}\right]=-\frac{\Omega S}{r} \\
& B_{\vartheta}=\frac{1}{r} \frac{\partial \tilde{S}}{\partial \vartheta}+\frac{1}{\sin \vartheta} \frac{\partial T}{\partial \varphi} \\
& B_{\varphi}=\frac{1}{r \sin \vartheta} \frac{\partial \tilde{S}}{\partial \varphi}-\frac{\partial T}{\partial \vartheta}
\end{aligned}
$$

using the abbreviation $\tilde{S}=\frac{\partial}{\partial r}(r S)$, see eq. (4).

With eqs. (8), (9), (11) and (26)-(28) we obtain the SH expansion of the field components

$$
\begin{aligned}
B_{r} & =\frac{1}{r} \sum_{n=1}^{n_{\max }} \sum_{m=0}^{n} n(n+1)\left[S_{n m}^{c} \cos m \varphi+S_{n m}^{s} \sin m \varphi\right] P_{n m}(\cos \vartheta), \\
B_{\vartheta} & =\frac{1}{r} \sum_{n=1}^{n_{\max }} \sum_{m=0}^{n}\left[\tilde{S}_{n m}^{c} \cos m \varphi+\tilde{S}_{n m}^{s} \sin m \varphi\right] \frac{\partial P_{n m}(\cos \vartheta)}{\partial \vartheta} \\
B_{\varphi} & =\frac{1}{r} \sum_{n=1}^{n_{\max }} \sum_{m=0}^{n}\left[\tilde{S}_{n m}^{s} \cos m \varphi-\tilde{S}_{n m}^{c} \sin m \varphi\right] \frac{m}{\sin \vartheta} P_{n m}(\cos \vartheta) .
\end{aligned}
$$

The magnetic flux $\mathbf{B}$ is continuous at $r=R_{\sigma}$ and the toroidal field is zero there like at any surface between a conductor and an insulator. The continuity of $B_{r}$ results that of the modes $S_{n m}^{c s}$ at $R_{\sigma}$ and the continuity of $B_{\vartheta}$ and $B_{\varphi}$ that of $\tilde{S}_{n m}^{c s}$ respectively. The continuity of $S_{n m}^{c s}$ gives the first boundary condition in eq. (13). The continuity of $\tilde{S}_{n m}^{c s}$ gives

$$
\left[S_{n m}^{c s}+r \frac{\mathrm{d} S_{n m}^{c s}}{\mathrm{~d} r}\right]_{R_{\sigma}^{+}}=\left[S_{n m}^{c s}+r \frac{\mathrm{d} S_{n m}^{c s}}{\mathrm{~d} r}\right]_{R_{\sigma}^{-}} .
$$

The representation of $B_{r}$ in eq. (29) is useful to find the relation between the geomagnetic Gauss coefficients, $g_{n m}, h_{n m}$, and the $S$-modes. The surface potential $V$ of $\mathbf{B}$ is usually given by

$$
V=R_{E} \sum_{n=1}^{n_{\max }} \sum_{m=0}^{n}\left(\frac{R_{E}}{r}\right)^{n+1} \lambda_{n m} P_{n m}\left(g_{n m} \cos m \varphi+h_{n m} \sin m \varphi\right),
$$

where $\lambda_{n m}$ are the Schmidt's normalization coefficients given by

$$
\lambda_{n m}=\left[\left(2-\delta_{0 m}\right) \frac{(n-m) !}{(n+m) !}\right]^{1 / 2}
$$


and $R_{E}$ is the mean Earth radius, respectively. Because of $\mathbf{B}=-\operatorname{grad} V$, we obtain for $B_{r}$

$$
B_{r}=\sum_{n=1}^{n_{\max }} \sum_{m=0}^{n}(n+1)\left(\frac{R_{E}}{r}\right)^{n+2} \lambda_{n m} P_{n m}\left(g_{n m} \cos m \varphi+h_{n m} \sin m \varphi\right) .
$$

Comparing (29) with (35), we obtain the equivalent potential coefficients

$$
S_{n m}^{c}=\frac{1}{n} \lambda_{n m} g_{n m}(t) R_{E}, \quad S_{n m}^{s}=\frac{1}{n} \lambda_{n m} h_{n m}(t) R_{E}
$$

to be continued to $r=R_{\sigma}$. At $r=R_{\sigma}^{+}$, the boundary condition (32) is due to the continuity of $d S / d r$, which can be computed according to $(36)$ as $-(n+1) r^{-1} S_{n m}^{c s}$, giving with the continuity of $S_{n m}^{c s}$ itself the second boundary equation in (13). 